05,12

\title{
Вертикальное смещение магнитооптической петли гистерезиса в магнитоплазмонном нанокомпозите
}

\author{
(ㄷ С.В. Томилин, В.Н. Бержанский, А.Н. Шапошников, А.Р. Прокопов, А.В. Каравайников, \\ Е.Т. Милюкова, Т.В. Михайлова, О.А. Томилина
}

Научно-исследовательский центр функциональных материалов и нанотехнологий, Физико-технический институт Крымского федерального университета им. В.И. Вернадского, Симферополь, Республика Крым

E-mail: tomilin_znu@mail.ru

Поступила в Редакцию 13 декабря 2018 г.

В окончательной редакции 19 августа 2019 г.

Принята к публикации 27 августа 2019 г.

\begin{abstract}
Представлены результаты синтеза и исследования магнитооптических (МО) свойств тонкопленочного магнитоплазмонного нанокомпозита, представляющего собой слой висмут замещенного феррит-граната $\mathrm{Bi}$ :YIG с плазмонными наночастицами Аu. При исследовании МО-свойств в процессе перемагничивания данного магнитоплазмонного нанокомпозита был обнаружен эффект вертикального смещения МО-петли гистерезиса относительно начала координат. Показано, что наблюдаемый эффект наиболее выраженно проявляется в окрестности условия локализованного плазмонного резонанса в системе металлических наночастиц, входящих в состав магнитоплазмонного композита.
\end{abstract}

Ключевые слова: тонкая пленка, наночастица, магнитооптика, плазмонный резонанс, нанокомпозит, эффект Фарадея.

DOI: $10.21883 /$ FTT.2020.01.48745.342

\section{1. Введение}

Получение эффективных магнитооптических (МО) систем и устройств для управления световыми пучками является одной из важных задач фотоники, плазмоники, сенсорики и т.д. [1-4]. Основное требование, предъявляемое к таким системам - получение максимального удельного МО-эффекта (эффекты Фарадея и Керра).

В настоящей работе в качестве МО системы исследуется магнитоплазмонный нанокомпозит состоящий из тонкой пленки висмут замещенного феррит-граната (Bi: YIG), содержащей самоорганизующиеся наночастицы Au. Введение в систему металлических наночастиц применяется для усиления МО-эффектов в окрестности условия локализованного плазмонного резонанса.

Подобные магнитоплазмонные системы неоднократно рассматривались различными авторами, в том числе и авторами данной работы [5-8]. При этом отмечалось, что на длине волны света, соответствующей частоте резонанса плазмонной подсистемы, наблюдается существенное усиление МО-эффекта Фарадея. Однако помимо усиления эффекта Фарадея, которое проявляется в виде увеличения полувысоты МО-петли гистерезиса, наблюдается так же дополнительный оптический эффект, не зависящий от знака внешнего магнитного поля. Данный эффект проявляется в виде вертикального, относительно начала координат, смещения МО-петли гистерезиса на постоянную величину $\Delta \theta$. Подобный эффект в неявном виде наблюдался ранее другими авторами, однако ему не уделялось должного внимания и часто смещение $\Delta \theta$ интегрально измерялось вместе с эффектом Фарадея и принималось за действительный результат фарадеевского вращения плоскости поляризации.

Экспериментальные исследования, проведенные в данной работе, а также анализ полученных результатов позволяют оценить закономерности возникновения данного эффекта и его свойства.

\section{2. Методы получения и исследования образцов}

В настоящей работе был синтезирован и исследован тонкопленочный магнитоплазмонный нанокомпозит $\mathrm{GGG} / \mathrm{Au}_{(\mathrm{NP})} / \mathrm{Bi}: \mathrm{YIG}$, где GGG - подложка из монокристаллического гадолиний-галлиевого граната с ориентацией плоскости (111); $\mathrm{Au}_{(\mathrm{NP})}$ - система самоорганизующихся золотых наночастиц (размещается непосредственно на подложке); Вi:YIG - тонкий слой висмут замещенного феррит-граната, нанесенного поверх золотых наночастиц. Общая структура магнитоплазмонной композитной системы представлена на рис. 1. Данный нанокомпозит был синтезирован методами вакуумного осаждения пленок с последующим отжигом.

\section{1. Синтез магнитоплазмонного нанокомпозита}

Синтез плазмонной подсистемы осуществлялся методом термического напыления Аu в вакууме. Испарение 


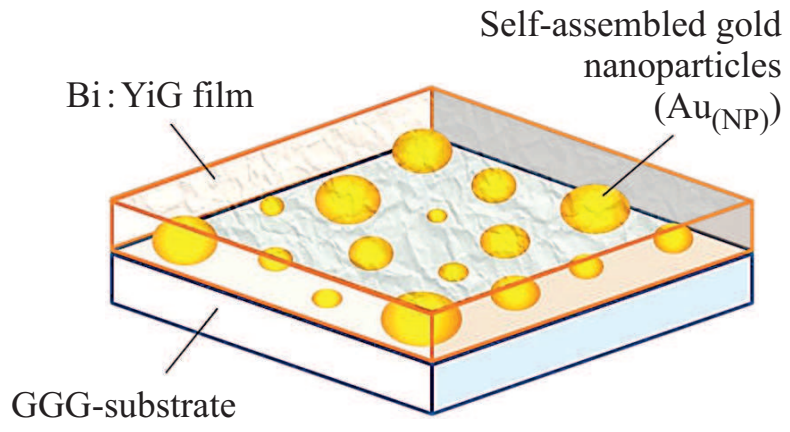

(Gallium-gadolinium garnet)

Рис. 1. Структурная модель магнитоплазмонной нанокомпозитной системы GGG/Au(NP) $/ \mathrm{Bi}$ : YIG.

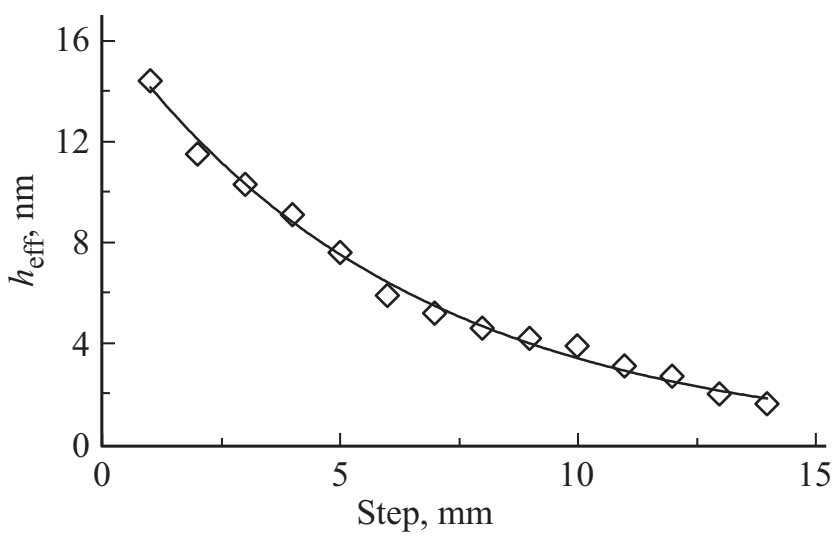

Рис. 2. Распределение $h_{\mathrm{eff}}$ пленки Аи вдоль градиента.

исходной загрузки Аu осуществлялось из вольфрамового тигля на прогретую до $150^{\circ} \mathrm{C}$ GGG-подложку. Для формирования системы самоорганизующихся наночастиц $\mathrm{Au}_{(\mathrm{NP})}$ исходное покрытие подвергалось отжигу на воздухе при температуре $950^{\circ} \mathrm{C}$ в течение $10 \mathrm{~min}$.

При формировании плазмонного покрытия использована методика напыления пленок с градиентом эффективной толщины $h_{\mathrm{eff}}[9]$. Такая методика позволяет в едином технологическом цикле при идентичных условиях синтезировать покрытие с переменной эффективной толщиной в определенном выбранном направлении вдоль образца. Так при выборе различных участков поверхности для исследований имеется возможность проводить сравнительный анализ влияния размерных эффектов на свойства и структуру покрытия.

Пленка $\mathrm{Au}$ после напыления имела градиент $h_{\mathrm{eff}}$, при котором максимальная эффективная толщина покрытия составляла $15 \mathrm{~nm}$, минимальная - $1 \mathrm{~nm}$. Профиль распределения $h_{\mathrm{eff}}(\mathrm{Au})$ вдоль градиента показан на рис. 2.

Напыление тонкой пленки феррит-граната Bi:YIG толщиной $100 \mathrm{~nm}$ осуществлялось методом ионнореактивного распыления мишени с номинальным составом $\mathrm{Bi}_{2.0} \mathrm{Gd}_{1.0} \mathrm{Fe}_{3.8} \mathrm{Al}_{1.2} \mathrm{O}_{12} \quad$ в газовой смеси $\operatorname{Ar}(25 \%)+\mathrm{O}_{2}(75 \%)$. После напыления пленка кристаллизовалась методом отжига на воздухе при температуре $680^{\circ} \mathrm{C}$ в течение $20 \mathrm{~min}$.

\section{2. Методы и методики исследования}

Исследование морфологии поверхности получаемых покрытий осуществлялось с использованием растрового электронного микроскопа (РЭМ, РЭМ-106 SELMI) и атомно-силового микроскопа (ACM, NTEGRA NT-MDT).

Для исследования спектров пропускания образца использован автоматизированный спектрофотометр КФК-3 с системой вывода и обработки сигнала на ПК. Спектральный диапазон исследования 400-980 nm. Для исследования покрытий вдоль градиента $h_{\mathrm{eff}}(\mathrm{Au})$ применялся специальный столик с микроперемещением для позиционирования образца.

Исследование МО-эффектов при циклическом перемагничивании образца осуществлялось с использованием автоматизированного монохроматического магнитополяриметра. Структурная схема магнитополяриметра показана на рис. 3.

Магнитополяриметр включает в себя источник когерентного монохроматического излучения (полупроводниковый лазер) с $\lambda=655 \mathrm{~nm}$. Оптическая часть измерительной схемы состоит из: поляризатора, задающего плоскость поляризации луча; магнитооптического модулятора для создания переменного сигнала на фоне общего светового шума; компенсатора, для выведения полезного сигнала в нуль и определения поворота плоскости поляризации; анализатора и фотодетектора.

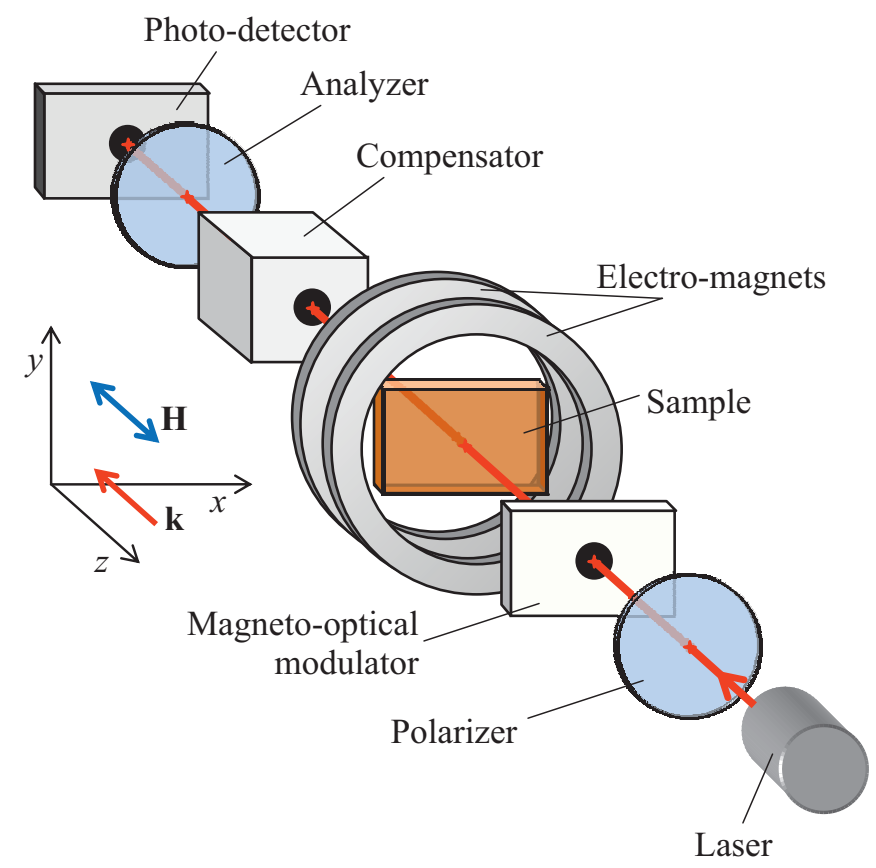

Pис. 3. Структурная схема магнитополяриметра. 

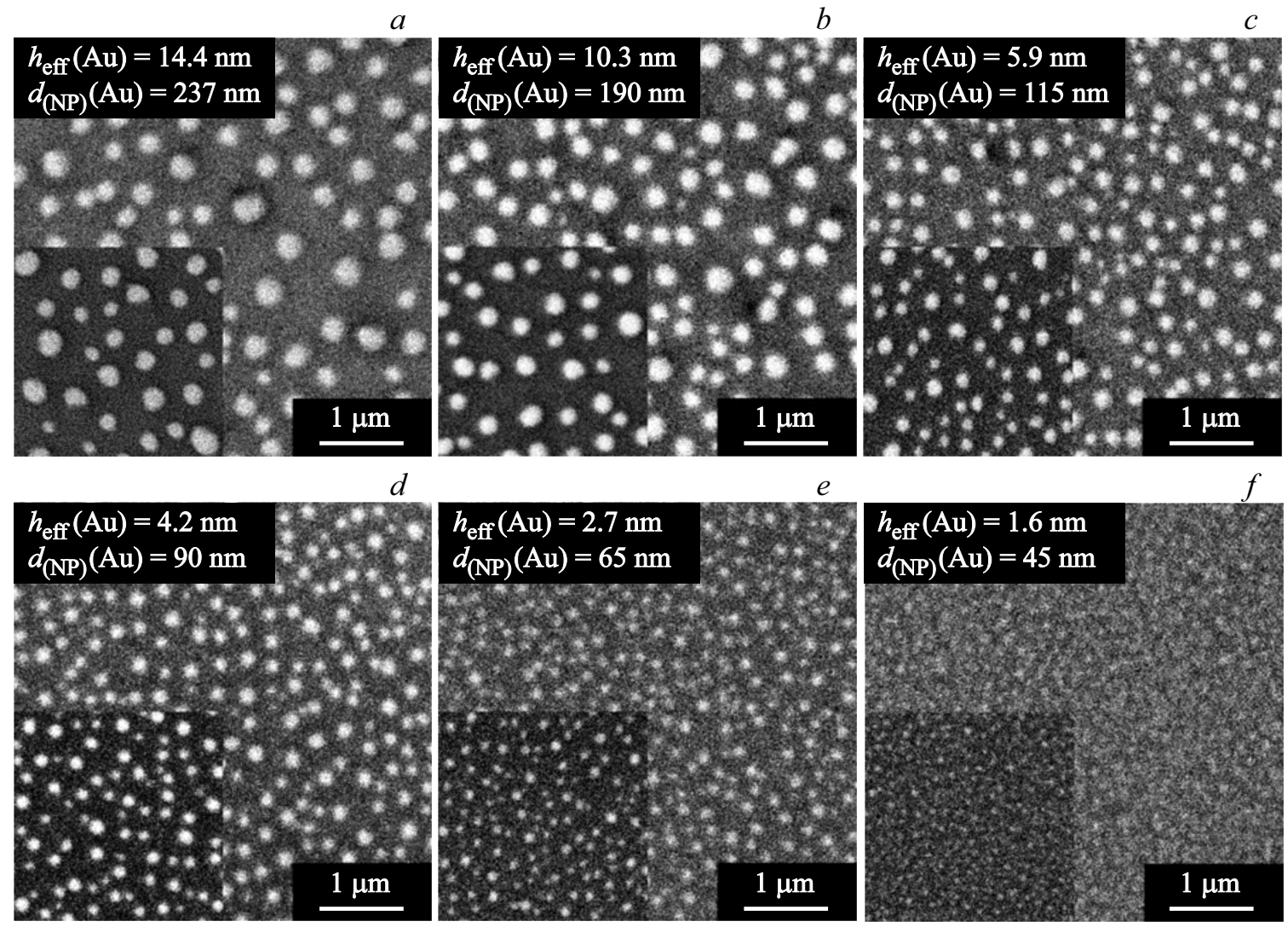

Рис. 4. Результаты РЭМ-исследования морфологии поверхности нанокомпозитной системы $\mathrm{GGG} / \mathrm{Au}_{(\mathrm{NP})} / \mathrm{Bi}: \mathrm{YIG}:(a-f)-$ участки образца, содержащие наночастицы Аu различного размера, на вставках показан участок поверхности с наночастицами GGG/Au $(\mathrm{NP})$ до напыления феррит-граната.
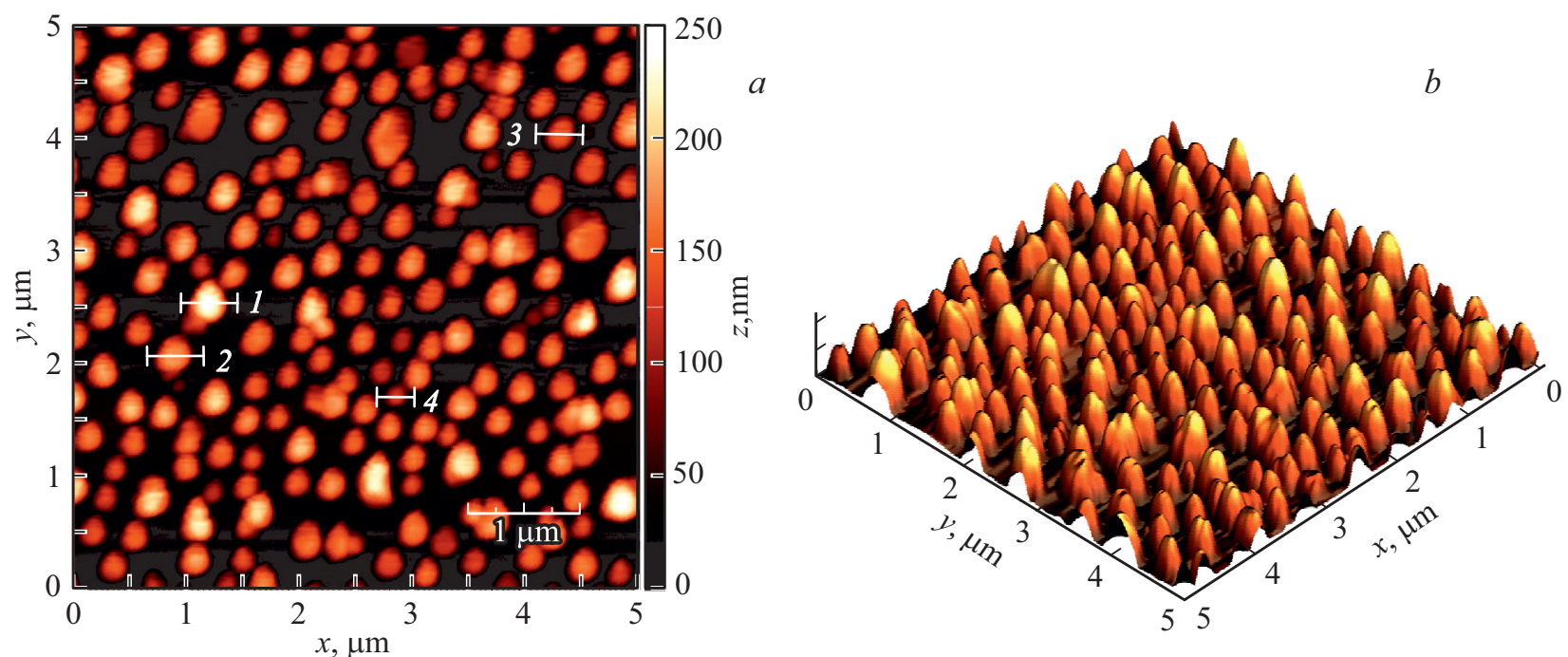

Рис. 5. АСМ-снимки рельефа золотых наночастиц $\mathrm{GGG}_{\mathrm{Au}}(\mathrm{NP}): a-2 \mathrm{D}$-диаграмма; $b-3 \mathrm{D}$-диаграмма.

Магнитная система магнитополяриметра состоит из электромагнитов с полыми сердечниками (на рис. 3 не показаны). Электромагниты создают магнитное поле в конфигурации, при которой вектор напряженности Н является коллинеарным с направлением волнового вектора излучения лазера.

\section{3. Результаты и их обсуждения}

\section{1. Структурные характеристики}

РЭМ-снимки морфологии поверхности нанокомпозита $\mathrm{GGG} / \mathrm{Au}_{(\mathrm{NP})} / \mathrm{Bi}: \mathrm{YIG}$ в различных участках вдоль гради- 


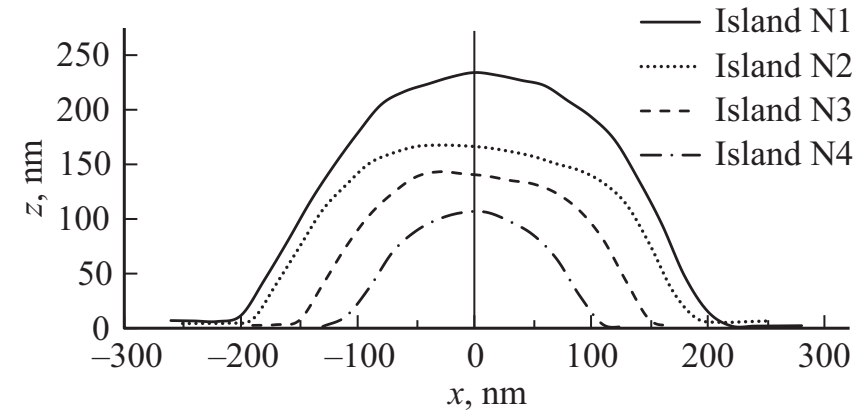

Pис. 6. Диаграммы сечений поперечного профиля наночастиц $\mathrm{Au}$ (ACM, NTEGRA NT-MDT).

ента $h_{\text {eff }}(\mathrm{Au})$ (содержат наночастицы золота с различным наиболее вероятным диаметром $\left.d_{(\mathrm{NP})}\right)$ представлены на рис. 4. На вставках к снимкам показаны участки поверхности с наночастицами $\mathrm{GGG} / \mathrm{Au}_{(\mathrm{NP})}$ до напыления феррит-граната. Как видно из представленных снимков после нанесения гранатовой пленки размеры частиц в среднем увеличились. Это свидетельствует о том, что металлические наночастицы находятся в диэлектрической оболочке из феррит-граната.

Для получения данных о пространственной форме $\mathrm{Au}$ наночастиц были проведены АСМ-исследования морфологии поверхности $\mathrm{GGG} / \mathrm{Au}_{(\mathrm{NP})}$ (рис. 5).

Как видно из представленной 3D-диаграммы наночастицы имеют форму объемных полуэллипсоидов с максимальной высотой до $235 \mathrm{~nm}$. Более подробно форму наночастиц можно исследовать на основании анализа сечений отдельных частиц различного размера. На рис. 5, а показаны линии сечений отдельных частиц, а соответствующие им диаграммы сечений показаны на рис. 6. Как видно из представленных диаграмм наночастицы имеют объемную форму близкую к полуэллипсоиду вращения со средним аспектным соотношением 0.5 (таблица). Таким образом, при анализе морфологии поверхности форму наночастиц полученных методом отжига можно аппроксимировать в виде „полукапель“ (полусфер вращения) с высокой долей достоверности.

Параметры наночастиц, полученные методом исследования сечений (рис. 6)

\begin{tabular}{|c|c|c|c|c|}
\hline $\begin{array}{c}\text { № } \\
\text { островка }\end{array}$ & $\begin{array}{c}\text { Диаметр } \\
\text { островка } \\
x, \mathrm{~nm}\end{array}$ & $\begin{array}{c}\text { Высота } \\
\text { островка } \\
z, \mathrm{~nm}\end{array}$ & $\begin{array}{c}\text { Аспектное } \\
\text { соотношение } \\
z / x\end{array}$ & $\begin{array}{c}\text { Среднее } \\
\text { аспектное } \\
\text { соотношение }\end{array}$ \\
\hline 1 & 400 & 234 & 0.58 & \multirow{4}{*}{$\sim 0.5$} \\
\hline 2 & 360 & 164 & 0.46 & \\
\hline 3 & 300 & 143 & 0.48 & \\
\hline 4 & 210 & 105 & 0.5 & \\
\hline
\end{tabular}

\section{2. Оптические свойства}

Для анализа оптических и плазмонных свойств магнитоплазмонного нанокомпозита $\mathrm{GGG} / \mathrm{Au}_{(\mathrm{NP})} / \mathrm{Bi}$ : YIG на всех стадиях его синтеза исследовалась спектральная зависимость коэффициента пропускания (рис. 7). Исследования проводились в различных точках вдоль градиента $h_{\text {eff }}(\mathrm{Au})$.

Так на рис. 7, $a$ представлены спектры пропускания пленки GGG/Au до отжига. В легенде указаны значения эффективной толщины $h_{\text {eff }}$ пленки на соответствующих участках. Как видно из представленных результатов, вид спектров пропускания соответствуют объемному поглощению $\mathrm{Au}[10]$, т.е. структура пленки на всех исследуемых участках является сплошной.

Данный вывод находит подтверждение и при анализе спектральных особенностей пропускания для различных

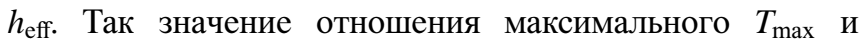
минимального $T_{\min }$ коэффициентов пропускания в зависимости от эффективной толщины $h_{\mathrm{eff}}$ меняется по экспоненциальному закону (рис. $7, b$ ), что свидетельствует об объемном характере поглощения в пленке.

На рис. 7, с и $e$ приведены спектральные зависимости коэффициента пропускания системы самоорганизованных золотых наночастиц $\mathrm{GGG} / \mathrm{Au}_{(\mathrm{NP})}$ (до напыления феррит-граната) и магнитоплазмонного нанокомпозита $\mathrm{GGG} / \mathrm{Au}_{(\mathrm{NP})} / \mathrm{Bi}$ : YIG. В легендах указан наиболее вероятный диаметр наночастиц $d_{(\mathrm{NP})}$ на исследуемом участке. На рис. 7, $d$ и $f$ показаны соответствующие графики спектрального смещения плазмонного резонанса при изменении $d_{(\mathrm{NP})}(\mathrm{Au})$.

B спектрах пропускания системы $\mathrm{GGG} / \mathrm{Au}_{(\mathrm{NP})}$ (рис. 7,c) можно выделить следующие особенности. Во-первых, на спектрах присутствуют четко выраженные минимумы, соответствующие поглощению энергии при резонансном возбуждении локализованных плазмонполяритонов $[3,6,11,12]$. Во-вторых, для крупных наночастиц наблюдается возбуждение двух типов резонансных колебаний (два резонансных „пика“): первый „голубой пик“ $(500-540 \mathrm{~nm})$ соответствует квадрупольным колебаниям, второй „красный пик“ - дипольным $[13,14]$. Следует отметить, что спектральное положение дипольного резонанса сильно зависит от размера наночастиц. При уменьшении размера частиц дипольный резонанс смещается в „синюю“ (коротковолновую) область спектра. Спектральное положение квадрупольного резонанса слабо меняется с изменением $d_{(\mathrm{NP})}$, а для малых наночастиц $\mathrm{Au}$ квадрупольный резонанс не наблюдается вовсе [15].

B спектрах нанокомпозита $\mathrm{GGG} / \mathrm{Au}_{(\mathrm{NP})} / \mathrm{Bi}: \mathrm{YIG}$ (рис. 7,e) можно отметить ряд особенностей вызванных присутствием слоя феррит-граната. Во-первых, в диапазоне длин волн 400-550 nm наблюдается сильное уменьшение коэффициента пропускания вызванное объемным поглощением пленки Вi:YIG. Во-вторых, наблюдается „сильное“ (порядка $100 \mathrm{~nm}$ ) спектральное смещение плазмонных резонансов в „красную“ область по сравнению со спектрами системы $\mathrm{GGG} / \mathrm{Au}_{(\mathrm{NP})}$, 

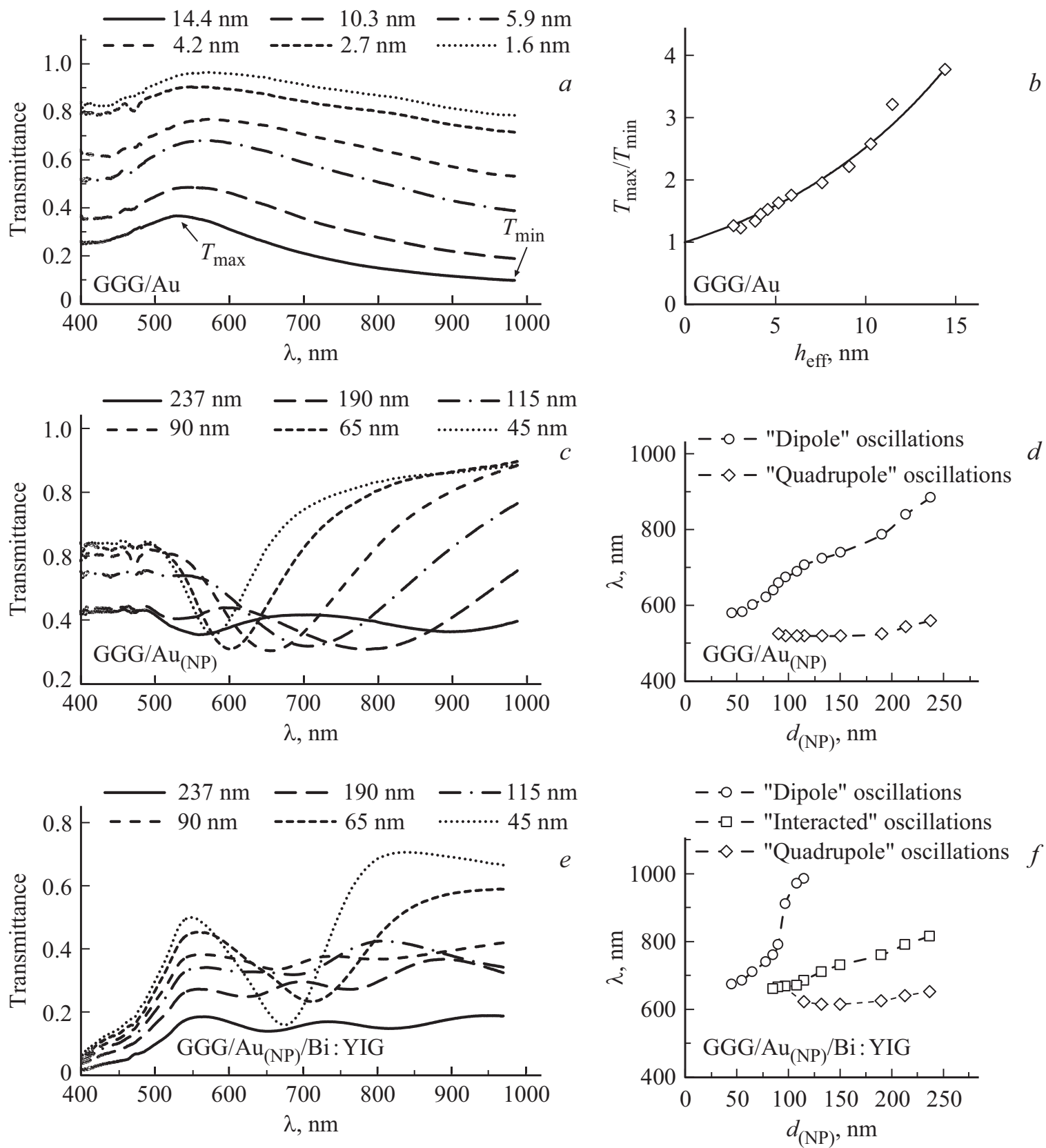

Рис. 7. Спектры пропускания нанокомпозита $\mathrm{GGG} / \mathrm{Au}_{(\mathrm{NP})} / \mathrm{Bi}: \mathrm{YIG}$ на различных стадиях синтеза: $a-\mathrm{GGG} / \mathrm{Au}$ (до отжига); $c-\mathrm{GGG} / \mathrm{Au}_{(\mathrm{NP})}$ (после отжига); $e-\mathrm{GGG} / \mathrm{Au}_{(\mathrm{NP})} / \mathrm{Bi}: \mathrm{YIG}$ (после напыления слоя феррит-граната); $b-$ зависимость от $h_{\mathrm{eff}}$ отношения максимального и минимального коэффициентов пропускания каждого спектра для образца $\mathrm{GGG} / \mathrm{Au} ; d, f-\mathrm{cmeщение}$ плазмонных пиков при изменении наиболее вероятного размера наночастиц $d_{(\mathrm{NP})}$ для образца $\mathrm{GGG} / \mathrm{Au}_{(\mathrm{NP})}$ и для нанокомпозита $\mathrm{GGG} / \mathrm{Au}_{(\mathrm{NP})} / \mathrm{Bi}: \mathrm{YIG}$.

что связано с влиянием магнито-диэлектрической гранатовой оболочки на металлических наночастицах. В-третьих, на спектрах присутствуют три „пика“ плазмонного резонанса (возбуждаются три типа резонансных колебаний). Два из них соответствуют дипольному и квадрупольному плазмонным резонансам. Подробный анализ параметров третьего резонансного пика показал, что по характеру зависимости от $d_{(\mathrm{NP})}$ он имеет характерное сходство со свойствами „квадрупольного“ резонанса, отличие составляют лишь резонансные частоты.
Таким образом, можно выдвинуть гипотезу о природе третьего пика, как резонанса на частоте колебаний связанных систем, состоящих из соседних квадруполей (связанные колебания). Подобная связь электромагнитной природы, очевидно, образовалась в результате нанесения слоя феррит-граната, диэлектрические и магнитные свойства которого и обусловили взаимодействие квадруполей. Кроме того, „квадрупольный“ резонанс и резонанс „связанных колебаний“ для частиц c $d_{(\mathrm{NP})}=80-120 \mathrm{~nm}$ сливаются в единый „пик 


\section{Магнитооптические эффекты}

На рис. 8 представлен характерный вид магнитооптических петель гистерезиса полученных методом детектирования угла вращения плоскости поляризации монохроматического когерентного плоскополяризованного света при перемагничивании образца нанокомпозитной системы $\mathrm{GGG} / \mathrm{Au}_{(\mathrm{NP})} / \mathrm{Bi}$ :YIG. Для сравнительного анализа на рисунке приведены петли, снятые на участках образца, содержащих плазмонные наночастицы с наиболее вероятным диаметром $\left(d_{(\mathrm{NP})}\right) 75 \mathrm{~nm}, 55 \mathrm{~nm}$ и без наночастиц (участок чистой гранатовой пленки GGG/Bi: YIG).

Как видно из представленного, для пленки GGG/Bi:YIG (без плазмонной подсистемы) MO петля гистерезиса имеет вид симметричный относительно начала координат. При этом максимальный угол фарадеевского вращения $\theta_{\mathrm{FR}(\max )}$ равен половине высоты петли и составляет по модулю $0.19^{\circ}$. При наличии плазмонной подсистемы МO петля гистерезиса для нанокомпозита $\mathrm{GGG} / \mathrm{Au}_{(\mathrm{NP})} / \mathrm{Bi}$ : YIG претерпевает изменения. Во-первых, изменяются высота и форма петли, что соответствует изменению полевой зависимости угла фарадеевского вращения. Во-вторых, петля испытывает смещение вдоль оси ординат на величину $\Delta \theta$. Таким образом, угол полного вращения плоскости поляризации $\theta_{\mathrm{TR}}$ складывается из двух углов: непосредственно угла фарадеевского вращения $\theta_{\mathrm{FR}}$ и величины смещения петли $\Delta \theta$. Зная угол полного вращения в полях насышения $+H_{S}$ и $-H_{S}$ можно определить значение максимального угла фарадеевского вращения

$$
\theta_{\mathrm{FR}(\max )}=\left[\theta_{\mathrm{TR}}\left(+H_{S}\right)-\theta_{\mathrm{TR}}\left(-H_{S}\right)\right] / 2
$$

и величины смещения МО-петли гистерезиса

$$
\Delta \theta=\left[\theta_{\mathrm{TR}}\left(+H_{S}\right)+\theta_{\mathrm{TR}}\left(-H_{S}\right)\right] / 2 .
$$

Анализ показал, что если из смещенной МО-петли гистерезиса нанокомпозита $\mathrm{GGG} / \mathrm{Au}_{(\mathrm{NP})} / \mathrm{Bi}: \mathrm{YIG}$ вычесть петлю гистерезиса чистой гранатовой пленки GGG/Bi: YIG, то получается прямая линия вида (рис. 9)

$$
\theta(H)_{\mathrm{GGG} / \mathrm{Au}_{(\mathrm{NP})} \mathrm{Bi}: \mathrm{YIG}}-\theta(H)_{\mathrm{GGG} / \mathrm{Bi}: \mathrm{YIG}}=\alpha H+\Delta \theta \text {. }
$$

где $\Delta \theta-$ и есть величина смещения МО-петли гистерезиса, определенная в (2), $\alpha H$ - линейный относительно поля член, определяющий усиление фарадеевского вращения.

Таким образом, в магнитоплазмонном нанокомпозите $\mathrm{GGG} / \mathrm{Au}_{(\mathrm{NP})} / \mathrm{Bi}$ :YIG наблюдаются два магнитооптических эффекта. Первый эффект есть нечетный относительно знака поля эффект Фарадея, который определяет форму магнитооптической петли гистерезиса. Второй эффект аддитивный к эффекту Фарадея линейный относительно поля и состоит из двух компонент: первая полевая компонента $\alpha H$ отвечает за изменение формы петли, а вторая $\Delta \theta$ не зависит от поля и определяет смещение МО-петли относительно начала координат.

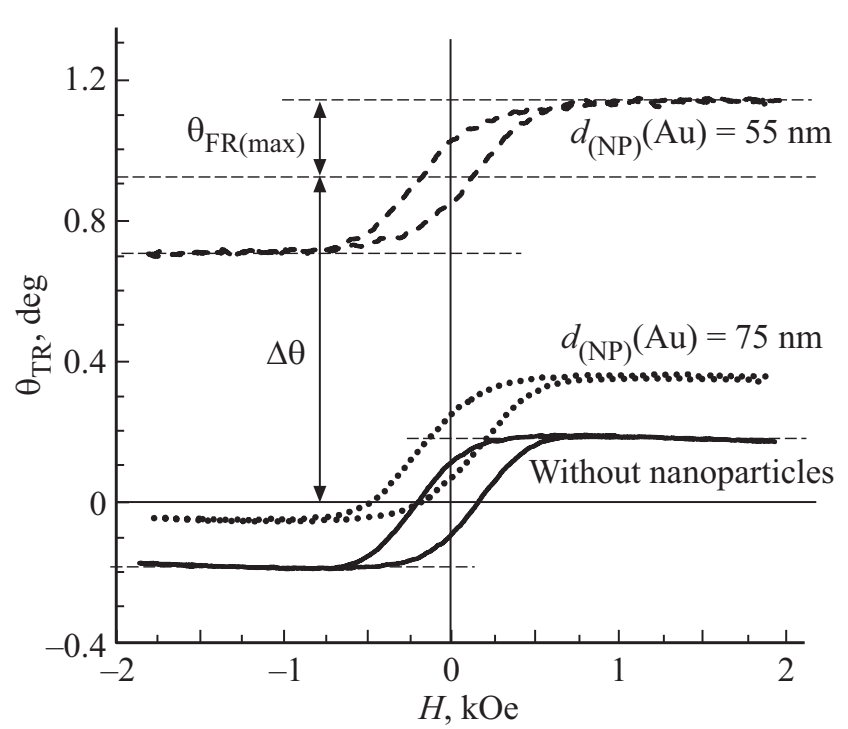

Рис. 8. Магнитооптические петли гистерезиса нанокомпозита GGG/Au ${ }_{(\mathrm{NP})} / \mathrm{Bi}$ :YIG на участках содержащих плазмонные наночастицы с наиболее вероятным диаметром $\left(d_{(\mathrm{NP})}\right) 75 \mathrm{~nm}$, $55 \mathrm{~nm}$ и без наночастиц.

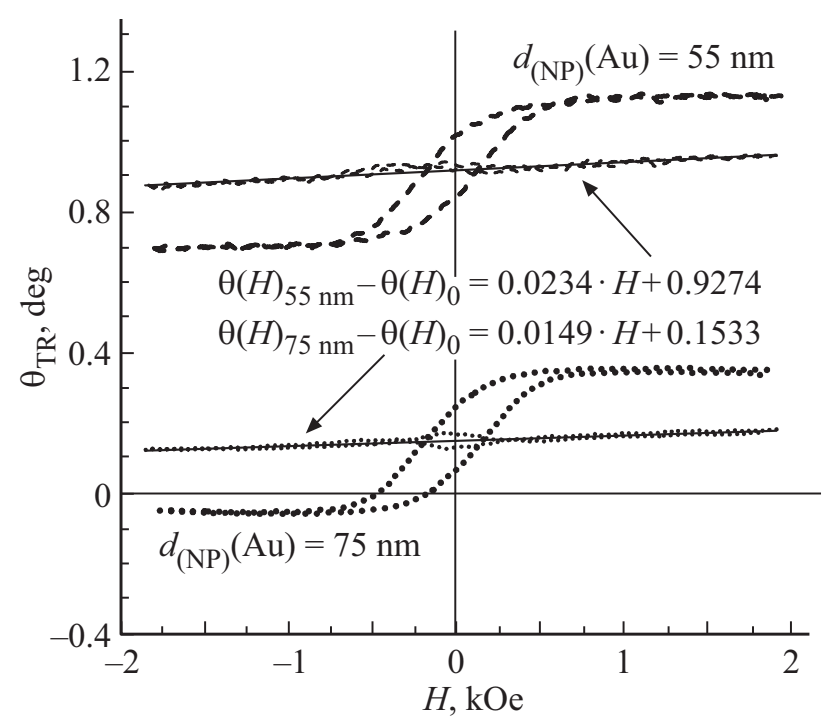

Рис. 9. Результат вычитания смещенной МО-петли для $\mathrm{GGG} / \mathrm{Au}_{(\mathrm{NP})} / \mathrm{Bi}: \mathrm{YIG}$ и несмещенной MO-петли для GGG/Bi:YIG (на фоне соответствующих смещенных петель).

На рис. 10 представлены графики зависимости от $d_{(\mathrm{NP})}(\mathrm{Au})$ для максимального угла фарадеевского вращения $\theta_{\mathrm{FR}(\max )}$ и смещения МО-петли $\Delta \theta$.

На представленных графиках видно, что угол фарадеевского вращения $\theta_{\mathrm{FR}(\max )}$ испытывает „сильный“ скачок в области $d_{(\mathrm{NP})}(\mathrm{Au})=40-50 \mathrm{~nm}$ (линия I на графике). Так угол $\theta_{\mathrm{FR}(\max )}$ вырос до $-0.27^{\circ}$, что по модулю более чем на $40 \%$ превышает аналогичное значение для чистой гранатовой пленки GGG/Bi: YIG (точка на графике $\left.d_{(\mathrm{NP})}=0 \mathrm{~nm}\right)$. Примечательно, что подобный скачок величина эффекта Фарадея испытывает в той области 


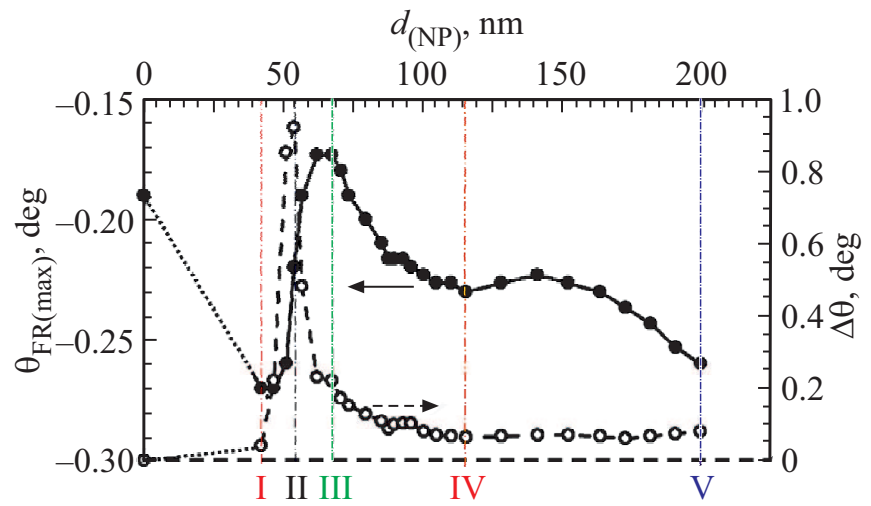

Рис. 10. Магнитооптические свойства нанокомпозита $\mathrm{GGG} / \mathrm{Au}_{(\mathrm{NP})} / \mathrm{Bi}: \mathrm{YIG}$ в зависимости от диаметра наночастиц золота $d_{(\mathrm{NP})}(\mathrm{Au})$ : закрашенные круги - угол фарадеевского вращения $\theta_{\mathrm{FR}(\max )}$, пустые круги - смещение МО-петли гистерезиса $\Delta \theta$.

образца, для которой резонансная частота возбуждения локализованных дипольных плазмон-поляритонов совпадает с частотой лазера магнитополяриметра (на длине волны $\lambda \approx 655 \mathrm{~nm}$ ).

На участке образца для $d_{(\mathrm{NP})}(\mathrm{Au})=60-70 \mathrm{~nm}$ (линия III на графике) наблюдается спад фарадеевского вращения вплоть до $-0.17^{\circ}$, что на $10 \%$ меньше аналогичного значения для чистой гранатовой пленки. То есть на данном участке наблюдается подавление эффекта Фарадея в пленке феррит-граната.

Далее по мере увеличения $d_{(\mathrm{NP})}(\mathrm{Au})$ величина фарадеевского вращения вновь увеличивается и имеет два максимума (по модулю), которые соответствуют резонансному совпадению частоты лазера и частот возбуждения квадрупольных (линия $\mathrm{V}$ на графике) и связанных (линия IV на графике) плазмон-поляритонных колебаний в наночастицах $\mathrm{Au}$.

Смещение МО-петли $\Delta \theta$ также испытывает значительный скачок в области $d_{(\mathrm{NP})}(\mathrm{Au})=55 \mathrm{~nm}$ (линия II на графике). Смещение петли при этом составляет $0.92^{\circ}$, что по модулю почти в 5 раз превышает величину угла фарадеевского вращения чистой пленки GGG/Bi:YIG и почти в 3.5 раза превышает максимальный эффект Фарадея в магнитоплазмонном нанокомпозите $\mathrm{GGG} / \mathrm{Au}_{(\mathrm{NP})} / \mathrm{Bi}:$ YIG. Для всех остальных исследованных участков образца значительные изменения величины $\Delta \theta$ не наблюдались, при этом смещение петли по мере увеличения $d_{(\mathrm{NP})}(\mathrm{Au})$ уменьшилось от 0.23 до $0.06^{\circ}$.

Примечательно, что пиковые возрастания эффекта Фарадея $\theta_{\mathrm{FR}(\max )}$ и смещения МО-петли гистерезиса $\Delta \theta$ не совпадают и наблюдаются на участках образца с различным значением $d_{(\mathrm{NP})}(\mathrm{Au})$. Однако следует отметить, что максимальное значение эффекта Фарадея $\theta_{\mathrm{FR}(\max )}=-0.27^{\circ}$ при $d_{(\mathrm{NP})}(\mathrm{Au})=42 \mathrm{~nm}$ соответствует минимальному значению $\Delta \theta=0.04^{\circ}$, зафик- сированному для магнитоплазмонного нанокомпозита $\mathrm{GGG} / \mathrm{Au}_{(\mathrm{NP})} / \mathrm{Bi}$ : YIG.

Отдельно следует оговорить, что в диапазоне $0<d_{(\mathrm{NP})}(\mathrm{Au})<42 \mathrm{~nm}$ отсутствуют данные об изменении $\theta_{\mathrm{FR}(\max )}$ и $\Delta \theta$ (на графике данный отрезок показан точками). Имеются лишь данные для точки $d_{(\mathrm{NP})}(\mathrm{Au})=0 \mathrm{~nm}$, то есть для чистой пленки ферритграната GGG/Bi:YIG, которая была получена как образец свидетель при напылении и последующем отжиге гранатового слоя в нанокомпозите $\mathrm{GGG} / \mathrm{Au}_{(\mathrm{NP})} / \mathrm{Bi}: \mathrm{YIG}$.

\section{3. Обсуждение обнаруженных эффектов}

Как было показано выше, на изменение МО-петли гистерезиса в магнитоплазмонном нанокомпозите $\mathrm{GGG} / \mathrm{Au}_{(\mathrm{NP})} / \mathrm{Bi}$ :YIG оказывают влияние два эффекта, общее действие которых может быть описано линейным уравнением вида (3).

Первый эффект описывается линейным относительно магнитного поля членом $\alpha H$ и отвечает за усиление эффекта Фарадея (увеличение высоты МО-петли гистерезиса). Данное усиление максимально проявляется на длинах волн, соответствующих плазмонному резонансу в плазмонной подсистеме нанокомпозита и многократно наблюдалось различными исследовательскими группами [5-7,16,17]. Очевидно, что данный эффект имеет исключительно плазмонную природу (определяется свойствами плазмонных наночастиц) и не может быть следствием проявления парамагнитных свойств подложки GGG (тем более, что во всех случаях подложка была одна и та же). Объяснение эффекта в рамках проявления диамагнитных свойств наночастиц $\mathrm{Au}$ также имеет серьезное противоречие. Так в статье показано, что при условии дипольного плазмонного резонанса усиление эффекта Фарадея (вклад линейного члена $\alpha H$ ) составляет более $40 \%$, а при условии квадрупольного резонанса - более $30 \%$ по сравнению с фарадеевским вращением в ферримагнитной пленке феррит-граната GGG/Bi:YIG без плазмонной подсистемы. Физическая природа данного эффекта, по нашему мнению, обусловленна локальным усилением электромагнитных полей в окрестности плазмонных наночастиц при условии возбуждения локализованного плазмонного резонанса $[18,19]$.

Второй из обнаруженных эффектов описывается в уравнении (3) аддитивной добавкой $\Delta \theta$, которая не зависит ни от знака, ни от величины приложенного магнитного поля и определяет величину вертикального смещения МО-петли гистерезиса. Подобный эффект смещения МО-петли авторы статьи [16] связывают с явлением поверхностного плазмонного резонанса (SPRinduced rotation) в анизотропной системе Аu наночастиц, однако отмечают, что на РЭМ-снимках данная анизотропия в явном виде не прослеживается. Следует отметить, что авторы настоящей статьи также не наблюдали структурной анизотропии на РЭМ-снимках морфологии поверхности $\mathrm{GGG} / \mathrm{Au}_{(\mathrm{NP})}$. 
Авторы статьи [17] связывают эффект смещения петли с явлением амплитудной и фазовой анизотропии пропускания плазмонной подсистемы для $s$ - и p-поляризованных волн. Очевидно, что описанная в [17] анизотропия пропускания, как и в [16], обусловлена структурной анизотропией плазмонной подсистемы. При этом в $[16,17]$ не выявлено наличие линейного полевого члена $\alpha H$, приводящего к усилению эффекта Фарадея.

Одной из причин возникновения вертикального сдвига МО-петли гистерезиса можно считать наличие в магнитоплазмонной композитной системе эффекта хиральности наноразмерных включений, или хирального расположения этих включений [20-25]. Эффект хиральности в свою очередь приводит к появлению кругового дихроизма (circular dichroism или CD) и как следствие к вращению плоскости поляризации. Однако следует отметить, что в перечисленных работах величина кругового дихроизма на наночастицах составляет единицы (иногда десятки) милиградусов, в то время как в настоящей статье авторами обнаружено смещение МО-петли (поворот плоскости поляризации) на 0.92 градуса, что превышает литературные данные более чем на порядок.

В статье [24] обнаружена полевая зависимость магнитного $\mathrm{CD}$ в наночастицах $\mathrm{Ag}$, в то время как в настоящей статье член $\Delta \theta$ определяющий величину вертикального смещения МО-петли гистерезиса от величины приложенного поля не зависит.

Согласно [23] высокие значения кругового дихроизма (сотни милиградусов) достигаются в пространственно неоднородных системах со скрещенными плазмонными диполями, однако в нашем случае наночастицы $\mathrm{Au}$ имеют сферическую форму и располагаются в плоскости подложки перпендикулярно к направлению волнового вектора падающего излучения, что исключает формирование скрещенных диполей. Второй способ получения большого CD состоит в формировании комплексов „хиральный лиганд-плазмонная наночастица“, что также исключено в рамках данной статьи.

Таким образом, вопрос о физической природе обнаруженных эффектов является до конца не раскрытым и формулируется в виде физических гипотез. Для более полного понимания сути данных эффектов необходимо проведение дальнейших исследований. Более полную информацию могли бы дать спектральные исследования эффекта усиления фарадеевского вращения и смещения МО-петли гистерезиса, что и будет являться дальнейшей целью наших исследований.

\section{4. Заключение}

На основании вышесказанного можно сделать следующие выводы.

При синтезе самоорганизующихся металлических наночастиц $\mathrm{GGG} / \mathrm{Au}_{(\mathrm{NP})}$ методом отжига сплошных пленок эффективная толщина покрытия влияет на размер получаемых наночастиц и, соответственно, на спектральное положение локализованного плазмонного резонанса. При этом в наночастицах возможно резонансное возбуждение двух типов резонансных плазмонов, дипольных и квадрупольных, испытывающих „красное“ смещение при увеличении размера резонирующих частиц.

B магнитоплазмонном нанокомпозите $\mathrm{GGG} / \mathrm{Au}_{(\mathrm{NP})} /$ $\mathrm{Bi}$ :YIG плазмонный резонанс испытывает дополнительное „красное“ смещение по сравнению со структурой $\mathrm{GGG} / \mathrm{Au}_{(\mathrm{NP})}$. При этом наблюдается появление третьего резонанса, обусловленного возникновением связанных колебаний соседних плазмонных квадруполей.

В нанокомпозите $\mathrm{GGG} / \mathrm{Au}_{(\mathrm{NP})} / \mathrm{Bi}: \mathrm{YIG}$ обнаружено усиление эффекта Фарадея по сравнению со структурой GGG/Bi:YIG в случае когда частота проходящего света совпадает с частотой плазмонного резонанса. При этом усиление эффекта Фарадея происходит на частотах как дипольного плазмонного резонанса, так и на частотах квадрупольного резонанса и резонанса связанных колебаний. Максимальное усиление эффекта Фарадея наблюдается в случае возбуждения дипольных резонансных плазмонов и составляет более $40 \%$.

В нанокомпозите $\mathrm{GGG} / \mathrm{Au}_{(\mathrm{NP})} / \mathrm{Bi}$ : YIG помимо усиления эффекта Фарадея наблюдается эффект вертикального смещения магнитооптической петли гистерезиса относительно начала координат. Данный эффект проявляется только при наличии плазмонной подсистемы и испытывает значительное усиление в области близкой к дипольному плазмонному резонансу. При этом усиление эффекта смещения МО-петли спектрально не совпадает с усилением эффекта Фарадея и проявляется на участках образца, где дипольный плазмонный резонанс несколько отличается по частотам от проходящего через образец света (при строгом совпадении частот наблюдаемый эффект смещения петли имеет минимальное значение). Максимальное смещение МО-петли гистерезиса в 5 раз превышает максимальный эффект Фарадея для пленки феррит-граната GGG/Bi:YIG и в 3.5 раза - для композитной системы $\mathrm{GGG} / \mathrm{Au}_{(\mathrm{NP})} / \mathrm{Bi}$ : YIG. При квадрупольном резонансе и резонансе связанных колебаний эффект наблюдается, однако не испытывает существенного усиления.

Обнаружено, что общее изменение МО-петли гистерезиса описывается аддитивной добавкой, линейно зависящей от поля. При этом свободный член $\Delta \theta$ в линейном уравнении добавки определяет вертикальное смещение МО-петли гистерезиса, а угловой коэффициент прямой $\alpha$ отвечает за усиление эффекта Фарадея.

\section{Финансирование работы}

Работа выполнена при финансовой поддержке Министерства науки и высшего образования Российской Федерации в рамках базовой части государственного задания (Проект № 3.7126.2017/8.9). 


\section{Конфликт интересов}

Авторы заявляют, что у них нет конфликта интересов.

\section{Список литературы}

[1] M. Inoue, A.V. Baryshev, A.B. Khanikaev, M.E. Dokukin, K. Chung, J. Heo, H. Takagi, H. Uchida, P.B. Lim, J. Kim. IEICE Trans. Electron 10, 1630 (2008).

[2] D.O. Ignatyeva, S.K. Sekatskii, A. N. Kalish, V.I. Belotelov. PIERS Proceedings, Prague, Czech Republic, 2296 (2015).

[3] K. Uchida, H. Adachi, D. Kikuchi, S. Ito, Z. Qiu, S. Maekawa, E. Saitoh. Nature Commun. | 6:5910 |DOI: $10.1038 /$ ncomms691 | www . nature.com/ naturecommunications.

[4] J. Bremer, V. Vaicikauskas, F. Hansteen, O. Hunderi. J. Appl. Phys. 11, 6177 (2001).

[5] Y. Mizutani, H. Uchida, Y. Masuda, A.V. Baryshev, M. Inoue. J. Magn. Soc. Jpn. 33, 481 (2009).

[6] H. Uchida, Y. Masuda, R. Fujikawa, A.V. Baryshev, M. Inoue. J. Magn. Magn. Mater. 321, 843 (2009).

[7] S. Tkachuk, G. Lang, C. Krafft, O. Rabin, I. Mayergoyz. J. Appl. Phys. 109, $07 \mathrm{~B} 717$ (2011).

[8] S.V. Tomilin, V.N. Berzhansky, A.N. Shaposhnikov, A.R. Prokopov, E.T. Milyukova, A.V. Karavaynikov, O.A. Tomilina. J. Phys.: Conf. Ser. 741, 012113 (2016).

[9] С.В. Томилин, В.Н. Бержанский, А.С. Яновский, О.А. Томилина. Поверхность. Рентгеновские, синхротронные и нейтронные исследования 8, 96 (2016).

[10] Sh. Wu, Yi. Cai, Ya. Bai, N. Liu, J. Zhu, Q. Liu, W. Chen. AsiaPacific Energy Equipment Engineering Research Conference (AP3ER 2015), 162 (2015).

[11] A. Ranjgar, R. Norouzi, A. Zolanvari, H. Sadeghi. Arm. J. Phys. 6, 198 (2013).

[12] P.B. Catrysse, Sh. Fan. Nano Lett. 10, 2944 (2010).

[13] K.L. Kelly, E. Coronado, L.L. Zhao, G.C. Schatz. J. Phys. Chem. B 107, 668 (2003).

[14] J. Rodriguez-Fernandez, J. Perez-Juste, F.J. Garcia de Abajo, L.M. Liz-Marzan. Langmuir 22, 7007 (2006).

[15] K. Kolwas, A. Derkachova. Opto-Electr. Rev. 4, 421 (2010).

[16] R. Fujikawa, A.V. Baryshev, J. Kim, H. Uchida, M. Inoue. J. Appl. Phys. 103, 07D301 (2008).

[17] A.V. Baryshev, H. Uchida, M. Inoue. J. Opt. Soc. Am. 9, 2371 (2013).

[18] A. Axelevitch, B. Apter, G. Golan. Opt. Express 214, 4126 (2013).

[19] K.L. Kelly, E. Coronado, L.L. Zhao, G.C. Schatz. J. Phys. Chem. B 107, 668 (2003).

[20] N. Engheta, D.L. Jaggard, M.W. Kowarz. IEEE Transact. Antennas Propagation 404, 376 (1992).

[21] A.J. Young, Ch.J. Serpell, J.M. Chin, M.R. Reithofer. Chem. Commun. 53, 12426 (2017).

[22] J. Cheng, E.H. Hill, Y. Zheng, T. He, Y. Liu. Mater. Chem. Front. 2, 662 (2018).

[23] A. Guerrero-Martinez, J. L. Alonso-Gomez, B. Auguie, M.M. Cid, L.M. Liz-Marzan. Nano Today 6, 381 (2011).

[24] M. Artemyev, R. Krutokhvostov, D. Melnikau, V. Oleinikov, A. Sukhanova, I. Nabiev. Proc. SPIE 8457, 845729 (2012). DOI: $10.1117 / 12.929860$.

[25] C. Noguez, I.L. Garzon. Chem. Soc. Rev. 38, 3, 757 (2009). DOI: $10.1039 / \mathrm{b} 800404 \mathrm{~h}$.

Редактор Д.В. Жуманов 